\title{
Introduction à l'histoire des Sames du sud
}

Introduction to South Saamis' history

Inledning till sydsamernas historia

\section{Guillaume Lestringant}

\section{OpenEdition}

Journals

Édition électronique

URL : https://journals.openedition.org/efo/3794

DOI : $10.4000 /$ efo.3794

ISSN : 2275-1947

\section{Éditeur}

INALCO

Édition imprimée

Date de publication : 1 janvier 2014

ISBN : 978-2-343-05394-3

ISSN : 0071-2051

\section{Référence électronique}

Guillaume Lestringant, « Introduction à l'histoire des Sames du sud », Études finno-ougriennes [En ligne], 46 | 2014, mis en ligne le 13 octobre 2015, consulté le 08 juillet 2021. URL : http:// journals.openedition.org/efo/3794 ; DOI : https://doi.org/10.4000/efo.3794

Ce document a été généré automatiquement le 8 juillet 2021.

\section{(c) (7) (8)}

Études finno-ougriennes est mis à disposition selon les termes de la Licence Creative Commons Attribution - Pas d'Utilisation Commerciale 4.0 International. 


\title{
Introduction à l'histoire des Sames du sud
}

\author{
Introduction to South Saamis' history \\ Inledning till sydsamernas historia
}

Guillaume Lestringant

\section{Introduction}

1 Avant de se lancer ${ }^{1}$ dans une histoire des Sames du Sud, il faut prendre le temps de déterminer de qui on parle. Donner une définition précise aux Sames du Sud est évidemment une tâche ardue, aussi ne me risquerai-je pas à décider ce qui caractérise l'appartenance au peuple same du Sud et ce qui n'est pas pertinent. Le Conseil Same, sorte de fédération de toutes les associations sames créée en 1956, a donné en 1986 sa propre définition des Sames :

We, Saami, are one people, united in our own culture, language and history, living in areas which, since time immemorial and up to historical times, we alone inhabited and utilised.

2 C'est cette définition, adaptée, que je vais m'efforcer d'utiliser : il sera question des gens ayant une culture commune du renne, et qui parlaient une langue same dans l'actuel espace de diffusion du same du Sud.

3 C'est pourquoi je vais m'intéresser dans un premier temps à l'époque et aux modalités d'apparition d'une communauté same du Sud en Jämtland, Härjedalen, Trøndelag et même un peu plus au sud. Dans un deuxième temps, je parlerai de l'histoire de cette communauté au cours de ce qui est pour nous le moyen âge, et particulièrement des raisons du passage d'une économie de chasse et de pêche à une économie pastorale. Puis il sera question du destin des Sames du Sud pris en tenailles entre les ambitions de la Suède, celles de la Norvège, et celles des paysans scandinaves, et enfin du long trajet jusqu'à leur reconnaissance légale. 
Pour des raisons de commodité, j'ai choisi d'employer dans leur langue d'origine les termes n'ayant pas d'équivalent aisé en français.

\section{La question des origines}

\section{Un aperçu historiographique}

5 Au cours des deux siècles écoulés, l'idée que l'on se faisait de l'origine des Sames a considérablement évolué. Dès 1821 , on était persuadé que les Sames étaient les descendants des peuples qui occupaient tout le Nord et l'Ouest de l'Europe à l'âge de pierre, et Sven Nilsson affirme, dans sa grande œuvre, Skandinaviska Nordens Urinvånare, ett forsök i comparativa Ethnographien, publiée en 1838 et 1843, que les Sames et le peuple des mégalithes ne font qu'un. Il s'appuie pour cela sur la craniologie, qui était à l'époque considérée comme une science sérieuse (Hansen \& Olsen 2007, p. 19-21). Mais dès les années 1860 , plusieurs archéologues se rendent comptent que les outils de l'âge de pierre sont en ardoise dans le Nord de la Scandinavie, alors qu'ils sont en silex dans le Sud. En 1867, Oluf Rygh en tire donc la conclusion qui semble s'imposer : il existe dès l'âge de pierre deux cultures archéologiques, donc deux peuples différents, en Scandinavie. Empreint des préjugés de l'époque, il associe le travail de l'ardoise aux Sames car, le matériau étant plus faible, il nécessiterait moins de travail que le silex, que Rygh associe donc aux Germains (Hansen \& Olsen 2007, p. 23).

6 Le dernier coup porté au rôle historique des Sames le sera par Yngvar Nielssen dans une allocution très médiatisée de décembre 1889 et dans une publication de 1891. Dans la première, il affirme que, de tous les noms de lieu sames qu'il a pu trouver en Norvège, le plus méridional était Nyamočokka, près du Gjevsjø en Nord-Trøndelag c'est à dire à la même latitude que Strömsund. Il en déduit donc que l'arrivée des Sames dans le Trøndelag est récente, postérieure au $\mathrm{XVI}^{\mathrm{e}}$ siècle. C'est une théorie qui a encore des partisans, dont le par ailleurs tout à fait correct Trøndelags historie. Dans la seconde, il s'attache à démontrer que les Sames ne peuvent pas être la culture de l'ardoise susmentionnée, et que par conséquent, il s'agit d'envahisseurs récents venus du NordOuest de la Russie (Hansen \& Olsen 2007, p. 25, Bergsland 1995, p. 112-113). Dans le demi-siècle qui suit, les historiens et les archéologues vont travailler de concert à consolider ce modèle d'une invasion récente de Sames venus de l'Est. En 1904, A. M. Hansen affirme que les Sames, des nomades du renne, sont arrivés

tardivement, et en tant qu'élément de peu d'importance (ma traduction).

7 Plus tard, en 1929, Gustaf Hällström explique la migration des Sames par une dégradation climatique à l'âge du fer préromain et romain, qui aurait dépeuplé le Nord de la Scandinavie et ouvert la voie aux envahisseurs. Enfin, en 1931 et 1935, K. E. Schreiner publie un ouvrage en deux tomes intitulé Zur Ostheologie der Lappen, où il définit le «type » same comme l'opposé presque parfait du «type » scandinave (Hansen \& Olsen 2007, p. 26-29).

8 Le renouveau est venu des travaux qu'ont menés les ethnologues dans les années 1960. En 1959, Povl Simonsen avance l'idée novatrice que l'ethnicité same ne serait pas un concept immuable, mais le résultat de processus sociaux et culturels. Cette idée sera précisée et généralisée par Fredrik Barth dans Ethnic Groups and Boundaries, publié en 1969. Il y affirme que l'ethnicité est un phénomène émergeant des contacts entre groupes sociaux. C'est sur ces bases théoriques que travaille Knut Ordner en 1983 : il 
développe un scénario complexe, dans lequel l'augmentation des contacts avec les Germains agriculteurs aurait poussé une partie des chasseurs-cueilleurs qui vivaient dans le Sud de la Finlande et sur la côte norvégienne à choisir d'adopter l'ethnicité germanique, tandis que d'autres auraient décidé de conserver leur mode de vie traditionnel, donnant ainsi naissance à l'ethnicité same (Hansen \& Olsen 2007, p. 30-36). Pierre Bourdieu, qui écrit en 1977, repris par Sián Jones en 1997, conteste l'aspect réfléchi de l'apparition d'une ethnicité : tant qu'un groupe humain est isolé, ses caractéristiques culturelles semblent aller de soi, et font naître des distinctions sociales au sein du groupe; c'est la mise en contact avec une communauté différente qui fait prendre conscience de la spécificité de ce fonctionnement et qui fait naître une distinction eux-nous plus importante que les distinctions internes (Hansen \& Olsen 2007, p. 37-38).

9 Pour Hansen et Olsen, il est logique de parler de Sames à partir de la fin du premier millénaire AEC (avant l'ère commune). En effet, ce premier millénaire AEC voit une augmentation importante des contacts entre les chasseurs-cueilleurs de Scandinavie et les métallurgistes de la culture d'Ananino en Russie, avec lesquels ils échangent des peaux contre des objets de métal. Parallèlement, une céramique de type Kjelmøy, caractérisée par une décoration en croisillons similaire à celle des haches en bronze de la culture d'Ananino, se généralise dans toute la zone, avant de disparaître dans les premiers siècles de l'ère commune, en même temps que les contacts avec la culture d'Ananino. L'apparition de cette culture archéologique commune est concomitante avec la germanisation des côtes norvégiennes et du Sud-Ouest du golfe de Botnie, et les deux chercheurs affirment que c'est en opposition à ces deux cultures que l'ethnicité same émerge chez les chasseurs-cueilleurs (Hansen \& Olsen 2007, p. 38-41).

10 Stig Welinder, quant à lui, considère leur datation comme trop ancienne. Il reconnaît l'existence d'un vaste ensemble de peuples en Finlande et en Suède, qui à partir de 1800 AEC utilise des fosses pour capturer les rennes et les élans sauvages, développe des relations commerciales avec les peuples de Russie pour importer du bronze, et utilise une poterie composée à 50-60\% d'amiante, ce qui la rend réfractaire (2008, p. 32-33). Cependant, il nie la possibilité de déterminer l'ethnicité, same ou autre, de ces populations. De même que les Germains ne constituèrent jamais une unité ethnique définie, mais étaient une construction intellectuelle des Romains, de même il serait importun de parler d'ethnicité à propos des chasseurs-cueilleurs de cette époque et de cette région (2008, p. 36-39). Au contraire, ce serait l'implantation autour du Storsjö d'une population à la culture radicalement différente, aussi bien matériellement qu'intellectuellement, de celle des chasseurs-cueilleurs, qui aurait conduit à l'émergence de deux ethnicités distinctes : d'une part les Jamts, agriculteurs, guerriers, enterrés sous des cairns, et fortement hiérarchisés; de l'autre les Sames du Sud, chasseurs-cueilleurs-pêcheurs, pacifiques, brûlés à leur mort selon une tradition spécifique, et plus ou moins égalitaires (2008, pp. 51-69). On peut cependant taxer cette vision de vouloir artificiellement apaiser le contentieux d'antériorité entre Jamts et Sames du Sud.

11 Si les modalités précises sont encore au stade des débats, il est au moins un point qui fait consensus: les Sames ne sont ni venus d'un autre endroit, ni les habitants immémoriaux de tout ou partie de la Scandinavie ; ils sont apparus il y a environ deux millénaires, lorsque des groupes de chasseurs-cueilleurs ayant entre eux plus ou moins 
une culture similaire ont développé une identité commune en opposition à d'autres groupes humains pratiquant entre autres l'agriculture.

\section{Le point de vue de la linguistique historique}

12 À partir de quand a-t-on parlé une langue same dans le Jämtland et le Trøndelag ? À cette question, il est plus facile de déterminer un terminus ante quam. Knut Bergsland s'est attaché dans les années 1980 à démonter la théorie de Yngvar Nielssen, en recensant l'ensemble des noms de lieux sames dans le Sud de la Suède et en étudiant les emprunts des différentes langues sames aux langues scandinaves. Sur le premier point, il trouve de nombreux lieux trahissant une implantation same ancienne dans le Trøndelag et le Jämtland, jusqu'à Finnfjellet près de Femunden, aux portes du Hedmark. Un peu plus au nord se trouve le village de Särvan, dans la commune de Härjedalen, dont le nom vient du same du Sud sarve «élan». Sur le second point, il définit une limite fort ancienne entre les Sames de Pite, Lule et du Nord d'une part, et les Sames d'Ume et du Sud d'autre part. Il fonde cette frontière sur l'existence de deux types de gievrie, le tambour chamanique traditionnel, dont la séparation se fait en Norvège à la hauteur de Rana, et sur des emprunts parallèles aux langues scandinaves. Par exemple, le proto-scandinave agju « tranchant, fil » a été emprunté vers le vi siècle en same du Nord sous une forme aivo, tandis qu'il a été emprunté en same du Sud sous une forme aavtjoe; ces deux formes ne peuvent pas provenir d'une même forme protosame. L'ensemble de l'exposé vise à démontrer qu'au $\mathrm{VI}^{\mathrm{e}}$ siècle de notre ère, le protosame était déjà divisé en au moins deux zones dialectales, dont aucune n'est la descendante de l'autre (Bergsland 1995, p. 1-39).

Si l'idée que le same est la langue autochtone de la Scandinavie est abandonnée, la date de l'arrivée du proto-same en Suède et en Norvège est plus controversée. Norbert Strade a affirmé en 1997 qu'une langue same était arrivée en Jämtland au plus tard entre 1000 et 500 AEC, et c'est l'avis que reprend Stig Welinder (2008, p. 48). Mais en 2006, Ante Aikio a publié un article qui suggère une date beaucoup plus récente. Dans son article, le chercheur étudie les emprunts du same, non au proto-scandinave ou à un de ses descendants, mais au proto-germanique. Il démontre qu'un nombre important de mots germaniques ont été empruntés à une époque qu'il qualifie de pré-same, car la langue n'avait pas encore subi les profonds changements de vocalisme qui font l'essentiel de la différence entre le proto-same et le proto-fennique. Par exemple, le proto-germanique *rauda- « rouge ", dont le sens a évolué pour désigner les morceaux de minerai de fer rougeâtre que l'on trouvait dans les tourbières - cf. le norrois rauði $i$ qui a ce sens-là - a été emprunté en pré-same sous la forme *rawta « fer ", ainsi qu'en finnois rauta «id.", avant de subir les changements vocaliques susmentionnés pour donner le proto-same ${ }^{*}$ ruovtē > same du Nord ruovdi. Si le mot avait été emprunté au norrois, on aurait trouvé une forme similaire à *ávdi. Le vocabulaire emprunté et le nombre important de mots concernés sont significatifs, car cela signifie que le protosame ne s'est réellement différencié du proto-fennique qu'à une date très tardive, manifestement à l'âge du fer ancien. Or on ne peut envisager que ces emprunts aient été réalisés uniformément sur un territoire allant du Cap Nord au Sud de la Finlande et au milieu de la Suède ; c'est donc que dans les premiers siècles AEC, le proto-same était encore parlé dans une zone restreinte de la Scandinavie (Aikio 2006, p. 39-42). 

revanche, il suggère qu'à l'époque pré-same, cette langue était parlée dans une vaste région lacustre allant de Tampere jusqu'au sud des lacs Onega et Ladoga, en passant par le Savo. En cela, il est suivi par Kallio 2009. Le Golfe de Finlande aurait alors pu faire une bonne zone de contact avec les marins parlant une langue germanique. Il fait également remarquer que dans la zone au sud des deux grands lacs devaient être parlés des idiomes plutôt para-sames, puisque les toponymes y ont des formes anormales (Aikio 2006, p. 42). On peut envisager qu'une langue pré-same très proche du protofennique ait été parlée aux abords et au sud des lacs Onega et Ladoga, avant de se répandre vers l'ouest et le Savo, région dans laquelle aurait eu lieu la transformation du système vocalique. chasseurs-cueilleurs parlaient un ensemble de langues ni ouraliennes, ni indo-européennes, et qui nous sont pour l'essentiel inconnues. On en retrouve cependant des traces importantes dans les diverses langues sames. De grands pans du vocabulaire same concernant la chasse, la pêche, les animaux et plantes locaux, ou encore le climat, n'ont pas d'étymologie ouralienne connue, de même qu'un certain nombre de toponymes. Si quelques caractéristiques de ces langues de substrat peuvent être aperçues - il y a une parenté entre certaines d'entre elles - le plus gros du travail d'identification reste encore à faire (Aikio 2006, p. 43-45).

16 S'il est acquis que les locuteurs du proto-same vivaient dans un espace réduit du Sud de la Finlande au début du premier millénaire AEC, il reste à définir quand, comment et pourquoi cette langue s'est étendue à au moins la moitié de la péninsule scandinave. Aikio apporte des éléments de réponse, en suggérant que la diffusion du proto-same soit concomitante à la diffusion de la poterie de type Kjelmøy vers $650 \mathrm{AEC}$, et que les motivations de cette expansion sont en lien avec le commerce des fourrures entre la Scandinavie en général et la région de la Volga (Aikio 2006, p. 45-47).

\section{L'apport possible de la génétique des populations}

Que peut apporter la génétique des populations à notre connaissance de la préhistoire des Sames? Assez peu, à vrai dire. Le lien entre une population, donc son génotype, et sa langue et sa culture est assez ténu, et les exemples de changement sont nombreux et connus. Cependant, dans ce cas précis, elle peut apporter plusieurs informations de détail intéressantes.

Pour ce qui est de l'ADN mitochondrial, qui ne se transmet que par les femmes, Ingman \& Gyllensten 2007 apporte un éclairage instructif. Les deux généticiens ont, en effet, étudié non la population same dans son ensemble, mais deux populations de Suède respectivement dans le domaine du same d'Ume et dans celui des sames de Pite et Lule - une population de Norvège et une de Finlande. Et voici ce que l'on peut en retirer.

- La population same est dans l'ensemble très homogène. Seuls se distinguent des autres les Sames d'Ume ayant adopté le mode de vie occidental, qui, sans surprise, ont une génétique très similaire aux Suédois. Elle est par ailleurs très proche de celle des Finnois, au point qu'une distinction est difficile à établir.

- Environ 80 \% des Sames appartiennent soit à l'haplogroupe V, soit à l'haplogroupe U5b. En cela ils sont des Européens des plus banals. Ces deux haplogroupes se retrouvent à des degrés divers dans toutes les populations d'Europe, car ils représentent les deux seules 
populations ayant survécu en Europe avant la dernière déglaciation. Ils seraient arrivés en Scandinavie respectivement vers 7600 AP (avant le présent) depuis la Sibérie et vers 6600 AP depuis la région de l'actuel Pays Basque.

- La quasi-totalité de la population same restante appartient à l'haplogroupe Z1, auquel appartiennent aussi les Finnois et les populations de langue finno-ougrienne de Russie occidentale. Les généticiens ont comparé leurs échantillons à d'autres, originaires de la région de Čeljabinsk au sud de l'Oural (sans doute des Maris), et sont arrivés à la conclusion que l'ancêtre commun le plus récent entre tous ces échantillons vivait vers 2700 AP. Cela signifierait que les locuteurs d'une langue finno-ougrienne n'ont quitté les rives de la Volga en direction du nord-ouest que dans la première moitié du premier millénaire AEC.

Quant à l'ADN du chromosome Y, qui se transmet uniquement par les hommes, l'état actuel de la science ne permet pas de tirer des conclusions aussi précises. On y retrouve les mêmes caractéristiques générales: deux populations arrivées après le dégel, l'une du sud-ouest (haplogroupe I1), l'autre du sud-est (haplogroupe R1a) ; des traces plutôt réduites de mélanges avec les locuteurs de langues germaniques (R1b3); enfin une origine génétique commune avec les locuteurs de langues finno-ougriennes du bassin de la Volga (N1c). Il n'y a pour l'instant pas de consensus sur la chronologie de l'arrivée de l'haplogroupe N1c en Scandinavie. Il existe, cependant, une différence importante avec les lignes matrilinéaires: là où l'haplogroupe mitochondrial Z1 représente une dizaine de pour-cent des Sames d'Ume, l'haplogroupe patrilinéaire N3 en représente près de la moitié, et jusqu'à $65 \%$ en Österbotten (Karisson 2006, Rootsi 2007). La raison de cette divergence reste encore à déterminer.

Enfin, les études qui portent sur l'ADN autosomal sont rares et n'ont pas encore apporté d'information concluante.

\section{De la chasse à l'élevage}

\section{L'âge de Vendel}

21 La situation humaine au début du $\mathrm{VII}^{\mathrm{e}}$ siècle dans la zone centrale de la péninsule scandinave est relativement claire. Une population de chasseurs-cueilleurs parlant vraisemblablement same est installée dans l'essentiel des zones boisées et un peu dans les montagnes. Parallèlement, il existe une population d'agriculteurs et d'éleveurs, parlant une langue germanique, qui est arrivée depuis plus ou moins longtemps : si les environs de Trondheim sont passés à l'agriculture depuis un millénaire, les colons commencent tout juste à occuper l'intérieur des terres; en Jämtland, les Scandinaves occupent les pourtours du Storsjö depuis deux siècles environ ; quant au Hedmark, il voit ses premières communautés agricoles apparaître entre le VI ${ }^{\mathrm{e}}$ et le viII ${ }^{\mathrm{e}}$ siècle autour de Hamar et de Brandval. Ce qui laisse de vastes territoires entièrement sous domination des chasseurs-cueilleurs : le Härjedalen, l'essentiel du Dalarna, le nord du Hedmark et les montagnes du Sør-Trøndelag. Les relations entre les deux communautés sont pacifiques et fondées sur le commerce. En effet, non contents d'être riches en gibier dont les fourrures intéressent les Germains, les bois servent aussi à produire du fer, lequel est utile aussi bien aux Sames pour leurs flèches et leurs lances qu'aux Germains, dont la société est militariste. La production de métal est même assez importante pour qu'une partie en soit exportée. Les Germains quant à eux produisent entre autres de la laine et des compléments à l'alimentation des Sames. 
$22 \mathrm{Au} \mathrm{VIII}{ }^{\mathrm{e}}$ siècle, la situation change dans le Jämtlands län. La production de métal baisse jusqu'à disparaître, et la population same du Härjedalen a sans doute aussi fortement décru: on ne trouve presque plus trace de fångstgropar, les fosses utilisées pour chasser le renne ou l'élan en grande quantité, et on n'a découvert qu'une poignée de tombes de cette époque. Le Härjedalen de l'époque se rapproche nettement du Dalarna, aussi bien dans les coutumes funéraires que dans l'abondance d'objets en métal que l'on retrouve dans les tombes: le Dalarna connaît à cette époque son pic de production de fer et exporte visiblement vers le nord. En revanche, d'après les individus trouvés dans les tombes du Härjedalen, il n'y a pas de différence notable dans leur habillement avec les Germains des rives du Storsjö. Le Jämtland est à ce moment-là plutôt intégré au réseau commercial et culturel du Trøndelag et du Medelpad (Welinder 2008, p. 72-73, 80-81). Dans le même temps, un changement culturel se fait jour parmi les Sames du Jämtland et du Härjedalen, qui aura une longue postérité : c'est, en effet, entre 500 et 900 qu'ils commencent à domestiquer quelques rennes pour en faire des animaux de trait et de bât, pour en consommer le lait, ou encore en guise d'appât pour la chasse (Welinder $2008: 81-83$, Hansen \& Olsen 2007, p. 204). Entre 600 et 800, ces hommes du renne commencent également à vivre dans des kåtor, une forme d'habitation qui a perduré jusqu'à nos jours (Welinder 2008, p. 105). Stig Welinder suggère, sans toutefois l'affirmer, qu'il aurait pu exister un sentiment d'appartenance ethnique commune entre ces populations des chasseurs-cueilleurs et celles vivant plus au nord. Il affirme en même temps qu'elles ne s'appliquaient pas l'auto-ethnonyme de "Sames " (2008, p. 81-83). Sur ce dernier point, je suis en désaccord avec l'archéologue : non seulement il n'apporte aucun argument, mais en plus le terme de "same » sous une forme ou sous une autre est attesté dans toutes les langues sames comme auto-ethnonyme et remonte à la période où le same et le finnois étaient encore indifférenciés.

\section{Un élargissement des horizons}

23 Les $\mathrm{IX}^{\mathrm{e}}$ et $\mathrm{X}^{\mathrm{e}}$ siècles constituent une période de changement social et économique pour les populations du milieu de la Scandinavie, caractérisée par l'intégration dans un réseau plus vaste, notamment de commerce à l'échelle européenne. Le IX siècle voit l'apparition d'États royaux dans le sud de la Scandinavie et au Danemark, avec lesquels les dirigeants en place vont devoir composer. Le Trøndelag, en raison de sa position stratégique, entre autres à proximité du puissant royaume de Hålogaland, et du fait de son absence de pouvoir central constitué, a très tôt suscité l'intérêt du royaume de Norvège en formation. Quant à la communauté des abords du Storsjö, elle semble avoir choisi de maintenir son indépendance vis-à-vis de ces royaumes en s'alliant avec d'autres provinces voisines ayant le même objectif (Welinder 2008, pp. 85-86). Au nord de la zone qui nous intéresse, un important réseau commercial se remet en place, qui importe des objets métalliques, essentiellement des bijoux, depuis l'Est, et exporte des peaux de grands mammifères. Ce réseau étend ses ramifications jusqu'aux Bulgares de la Volga (Hansen \& Olsen 2007, p. 136).

Ces royaumes émergents ont parfois également des rapports directs avec les Sames, ou du moins avec ceux qu'ils appellent les Finnar. Ainsi, la Saga de Haraldr Hárfagr, qui se déroule au tournant des deux siècles, raconte comment il a épousé une certaine Snæfriður, fille de Svási «Roi des Finnar », qui vivait à proximité de Dovre (Hansen \& Olsen 2007, p. 108). Ce Svási était-il Same, au moins linguistiquement? Rien ne permet 
de l'affirmer. En effet, à l'origine, le terme de finnar, apparenté à finna "trouver ", est une dénomination générique pour ceux qui trouvent leur nourriture, c'est à dire pour des chasseurs-cueilleurs, par opposition aux Germains qui la produisent. Ce n'est que plus tard que le terme n'a plus désigné que des locuteurs d'une langue finno-ougrienne. Or le nom de Svási ne paraît pas être same: une recherche dans le dictionnaire étymologique du same, Álgu ${ }^{2}$, montre que le same du Sud ne compte qu'une trentaine de mots commençant par sv-, qui sont pour l'essentiel des emprunts aux langues scandinaves. Au contraire, le nom est attesté dans d'autres textes norrois, et doit sans doute être rapproché du terme svásligr " adorable, merveilleux ", attesté dans l'Edda.

$\mathrm{Au}$ milieu de ce contexte globalisant, le Jämtland et le Härjedalen évoluent singulièrement. La production de fer dans les confins boisés du Härjedalen, ainsi que le nombre de fångstgropar, recommencent à augmenter. Et dans le même temps, les études palynologiques menées dans les tourbières du Stor-Mittåkläppen ${ }^{3}$ montrent qu'au plus tard au $\mathrm{x}^{\mathrm{e}}$ siècle, les versants de la montagne étaient utilisés comme pâturage, et les plaines en contrebas portaient des cultures (Welinder 2008, p. 84). Il pourrait s'agir d'un afflux de populations germaniques: le mythe de fondation de la province place l'arrivée des premiers Scandinaves à cette époque ; cependant, il s'agit là d'un récit de fondation stéréotypé, et aucun élément extérieur ne vient le corroborer. Il pourrait aussi bien s'agir d'un groupe same qui aurait adopté le mode de vie des Jamts. En effet, la principale caractéristique du Jämtland et du Härjedalen à cette époque est qu'il est impossible de distinguer clairement, dans les restes archéologiques, entre les chasseurs-cueilleurs et les agriculteurs. Il y a eu vraisemblablement une créolisation ethnique, tant et si bien que le paysage archéologique est constitué d'un continuum culturel, qui ne permet pas de séparer ne serait-ce que deux groupes homogènes : ceux qui se font enterrer à proximité des fermes et ceux qui se font enterrer dans les bois portent tous à peu près les mêmes vêtements, utilisent des objets similaires, et ont des coutumes funéraires difficilement discernables (Welinder 2008, p. 105-107, 101). Même les coutumes religieuses montrent un mélange important! Autour du Storsjö ont été excavés plusieurs lieux de culte, construits autour d'un bouleau, sous lequel les habitants d'alors ont enterré des restes d'animaux. Or il s'agit très majoritairement de restes d'ours, qui est un animal central dans la mythologie same, mais beaucoup moins important dans la mythologie scandinave (Welinder 2008, p. 90-91).

Une confusion similaire existait peut-être également dans le Nord-Trøndelag : à Verdal a été découvert un cimetière $\mathrm{du} \mathrm{x}^{\mathrm{e}}$ siècle, manifestement norrois, mais où un nombre important de tombes contenaient des bijoux sames (Hansen \& Olsen 2007, p. 106-107).

\section{Les derniers païens}

Cette belle harmonie va éclater en raison même des contacts nouvellement créés avec les royaumes suédois et norvégiens apparus depuis peu. Dès les années 1020, les rois de Suède et de Norvège commencent à exiger des dirigeants jamts qu'ils leur payent un tribut. Parfois les Jamts acceptent, parfois ils envoient les émissaires sur les roses. Ils ont cependant conscience qu'il faut faire des concessions pour maintenir de bonnes relations commerciales, indispensables à la survie économique de la province. Ils font alors le choix de se convertir au catholicisme (Welinder 2008, p. 125). Cela se fait progressivement au cours des $\mathrm{XI}^{\mathrm{e}}$ et $\mathrm{XII}^{\mathrm{e}}$ siècles. Au plus tard en 1050, les Jamts sont convertis à titre privé, ainsi qu'en témoigne la disparition brutale de la crémation et de 
l'enterrement des restes brûlés sous un tertre, comme cela se pratiquait depuis leur arrivée en Jämtland. Puis c'est la société qui se christianise, par l'apparition des premières églises à la fin du siècle et l'érection vers 1080 de la pierre runique de Frösön qui clame la christianisation du Jämtland. Mais la communauté nouvellement chrétienne ne fait pas encore partie de la grande maison catholique : Adam de Brême, qui écrit vers 1090, ne connaît pas l'existence du Jämtland lorsqu'il décrit l'état religieux de la Scandinavie. C'est au cours du XII siècle que les groupes de fidèles s'organisent en paroisses, puis se soumettent à un archevêché : Uppsala pour le Jämtland, Trondheim pour le Härjedalen (Welinder 2008, p. 109-113, 120-122, 135).

De cette conversion au catholicisme naît une aversion envers les Sames restés païens. Plus au sud, l'Eidsivatingslov et la Borgartingslov, les lois écrites au $\mathrm{xl}^{\mathrm{e}}$ siècle qui s'appliquaient dans les territoires au nord d'Oslo - une centaine de kilomètres interdisent formellement de se rendre chez les Finnar pour connaître l'avenir ou profiter de leur sorcellerie (Hansen \& Olsen 2007, p. 109). Dans le même ordre d'idée se trouve l'Historia Norvegiae, écrite en Norvège vers 1090, qui parle abondamment des Sames. Auparavant, et depuis Tacite, les Finnar étaient toujours décrits comme primitifs et sous-développés ; là apparaît pour la première fois l'idée que les Sames sont mauvais, vraisemblablement en raison de leur paganisme, plutôt que pour des motifs ethniques (Welinder 2008, p. 132-133). C'est en grande partie pour cette raison qu'à partir du XI $\mathrm{e}^{\mathrm{e}}$ siècle, les élites jamts cessent d'exploiter les confins de leurs territoires par l'intermédiaire des Sames, et se constituent à la place un réseau de fermes dans lesquelles ils installent des esclaves chasés ${ }^{4}$ (Welinder 2008, p. 126).

En conséquence, les Sames ont dû se constituer leur propre réseau économique, indépendant des Jamts. En même temps que la république de Novgorod, en s'étendant vers les lacs Ladoga et Onega, a pris le contrôle du commerce avec la Volga (Hansen \& Olsen 2007, p. 137), ce réseau s'est étendu vers le Sud : les broches, bijoux et pendentifs que l'on trouve dans les tombes sames du Jämtland et du Härjedalen sont très similaires à ceux que l'on retrouve en Finlande et en Russie. C'est ainsi qu'émerge une culture matérielle proprement same, qui se recentre en partie sur ce qui faisait autrefois sa spécificité : par exemple, certains Sames se mettent à déposer des bois de renne ou d'élan sur d'anciennes tombes à la mode païenne (Welinder 2008, p. 129). Cette nouvelle culture same peut être décrite comme suit, d'après ce que l'on a retrouvé dans les sites de Vivallen, Härjedalen, occupé entre 900 et 1300, et de Sösjön, dans le sameby de Njaarke, occupé entre 1000 et 1300. Ils vivent dans des kåtor de quatre ou cinq mètres de diamètre, avec en leur centre un foyer rectangulaire délimité par des pierres. La couverture est constituée d'une charpente de bois recourbé recouverte d'écorce de bouleau et de tourbe. Les habitants mangeaient essentiellement du renne, mais également de la chèvre ou du mouton, que manifestement ils élevaient, ainsi que du poisson. L'élevage de moutons ou chèvres est peut-être lié à leur besoin d'habits en laine. Ils fondaient du fer, essentiellement pour se fabriquer des pointes de flèche. On trouve toujours des traces d'un élevage limité de rennes, sous la forme d'enclos pour la traite (Welinder 2008, p. 128, Hansen \& Olsen 2007, p. 105). Après leur mort, ils étaient enterrés habillés, enveloppés dans de l'écorce de bouleau, mais sans être brûlés, contrairement à ce qui se faisait auparavant, ainsi qu'ils ont été retrouvés dans les cimetières de Vivallen et de Långön (Welinder 2008, p. 118). Un mort particulièrement intéressant est celui retrouvé dans la tombe 9 de Vivallen, datée du XII siècle. Il s'agit d'un vieil homme, portant une luxueuse ceinture inspirée de celle des nomades de la 
steppe. Il porte un collier de perles de quartz et, à sa ceinture, pendent une nålhus, un tube de bronze qui servait à conserver des aiguilles, ainsi que des morceaux de bronze brillant. Il est intéressant de noter que ces attributs seront par la suite associés aux femmes, mais que ce n'était manifestement pas encore le cas (Welinder 2008, p. 131).

Les Sames n'étaient cependant pas entièrement coupés du monde scandinave. Les rois de Norvège travaillaient en effet à imposer leur domination dans le Nord. Afin de faire concurrence à la République de Novgorod, les rois imposent dès le $\mathrm{xI}^{\mathrm{e}}$ siècle que le commerce avec les Sames devienne un monopole royal, ce qui se traduit par l'apparition de monnaies scandinaves dans les tombes sames (Welinder 2008, p. 133, Hansen \& Olsen 2007, p. 138-141). Et au XII e siècle, ils augmentent leurs contacts avec les Jamts, jusqu'à ce que Sverrir Sigurðarson, qui avait besoin d'une base arrière pour attaquer Trondheim, leur impose sa domination en 1178, après la bataille de Sunne, au sud-ouest d'Östersund (Welinder 2008, p. 136). Les contacts culturels continuent à exister, même de manière plus réduite. On trouve des restes d'une chasse au renne pratiquée par les Jamts (Welinder 2008, p. 137). À Øvre-Rendalen, dans le Hedmark, il a été découvert trois «marteaux de Thor » décorés sur une face à la mode norroise, sur l'autre à la mode same. Et toujours dans le Hedmark, de nombreuses tombes norroises contiennent des anneaux de type oriental (Hansen \& Olsen 2007, p. 106-109). On peut se demander s'il ne s'agit pas là d'amulettes données par les nåejtie, les chamans sames, ceux-là même que les chrétiens de la région d'Oslo ne sont pas censés aller voir.

\section{Les signes précurseurs du pastoralisme}

31 Se pose désormais la question de savoir quand, comment, et pourquoi les Sames sont passés d'un mode de vie de chasseurs-cueilleurs à un mode de vie pastoral. En effet, au point où l'on en est, les Sames du Sud ne pratiquent pas le pastoralisme, malgré leurs petits troupeaux : on ne peut parler de pastoralisme qu'à partir du moment où les humains exercent un contrôle sur la reproduction des animaux (Hansen \& Olsen 2007, p. 204). La question du quand et du pourquoi est assez claire, mais on y reviendra dans la partie suivante. C'est le comment qui est plus débattu. Des théories un peu anciennes supposent que les Sames les plus pauvres auraient, dans un cadre de disette, commencé à utiliser leurs quelques rennes domestiqués à des fins de nourriture, puis que, petit à petit, l'augmentation du nombre de pasteurs aurait conduit à un basculement général par une sorte de cercle vicieux. Mais cette explication ne convient pas à Hansen \& Olsen, car selon eux, l'organisation du travail dans un grand troupeau ne saurait se mettre en place à une échelle aussi individuelle. De plus, elle n'explique pas pourquoi la propriété commune des bêtes serait devenue individuelle (2007, p. 210-211). En effet, entre une société de chasseurs-cueilleurs, où les marques de propriété sont apposées sur les armes - comme cela a été retrouvé à Kjelmøy - de manière à déterminer qui a tué l'animal et en déduire le statut social inhérent, et une société pastorale, où la marque de propriété est apposée sur les animaux vivants, sous la forme d'entailles dans les oreilles, il y a nécessairement eu un changement profond de mentalité (Hansen \& Olsen 2007, p. 208-209).

Les deux auteurs proposent un scénario différent et très élégant. L'intégration dans une économie à plus grande échelle a conduit à augmenter la production et donc la quantité de bêtes chassées et abattues. Pour arriver à un tel résultat, la technologie a évolué à partir du XIII ${ }^{e}$ siècle, avec notamment l'apparition de clôtures de bois longues de 
plusieurs kilomètres qui seraient à guider les rennes vers les fosses de capture, les fångstgropar. Ces clôtures, que les Sames du nord appellent vuopmanat, seront réutilisées telles quelles pour délimiter les gigantesques enclos tels qu'ils existent encore de nos jours. Mais cette nouvelle technologie nécessitait un surcroît d'organisation. Les meilleurs chasseurs, qui jusqu'à présent se voyaient récompensés essentiellement par la gloire, se sont trouvés dans une position dominante, car ils organisaient la chasse. Ils ont ainsi créé une petite élite, dotée d'un réel pouvoir politique, et qui vers le $\mathrm{xv}^{\mathrm{e}}$ siècle s'est accaparé le droit de commercer avec les Scandinaves. L'apparition d'un droit de propriété sur les animaux vivants s'est sans doute faite pour deux raisons concomitantes. D'une part, cette élite a sans doute imposé comme un de ses privilèges la propriété des animaux qui étaient capturés vivants pour servir au trait ou pour le lait, laquelle se serait par la suite étendue aux animaux sauvages. D'autre part, le droit norvégien étant dominé par la notion de propriété, les chefs sames auront sans doute voulu éviter que d'autres ne viennent empiéter sur leurs territoires de chasse, en décrétant les troupeaux sauvages comme leur propriété. C'est pendant cette période que la sijte est devenu une entité territoriale et plus seulement sociale (Hansen \& Olsen 2007, p. 211-214, 140).

\section{Entre le marteau de Thor et l'enclume}

\section{L'impôt comme moyen d'expansion}

Comment des relations d'ordre commercial se sont muées en un système d'imposition par les États royaux, cela nous est encore pour l'essentiel inconnu. Toujours est-il qu'à partir du XIV siècle apparaissent dans les sources des gens nommés les birkarlar. Agissant dans toute la partie nord de la Suède, ce sont des marchands qui ont un droit exclusif de commerce et d'imposition sur les Sames, et ils sont eux-mêmes taxés pour cela par les rois de Suèdes. Pour régler les conflits entre eux et les Sames, les rois nomment des lappfogdar - le premier connu est mentionné à Pite en 1424. Plus au sud, cependant, les Sames de la paroisse d'Umeå et d'Ångermanland sont taxés directement par le lappfogde de leur région respective ${ }^{5}$. Pour ce qui est de la Norvège, qui à cette époque inclut encore le Jämtland et le Härjedalen, ce sont les seigneurs féodaux qui prélèvent l'impôt pour le compte du roi. C'est la situation telle qu'on la rencontre pendant toute la première moitié $\mathrm{du} \mathrm{XvI}$ siècle.

En théorie, du moins. Car la Suède va utiliser tout au long des $\mathrm{XVI}^{\mathrm{e}}$ et $\mathrm{XVII}^{\mathrm{e}}$ siècles l'imposition des Sames comme un moyen de justifier ses prétentions territoriales. En 1553, Gustav Vasa réforme le fonctionnement de l'imposition des Sames dans son royaume : il se passe désormais des birkarlar, et l'impôt dans les lappmarkar n'est plus levé que par les lappfogdar. Sauf que ces lappmarkar n'ont pas la même étendue selon que l'on se place du point de vue suédois ou norvégien. L'exemple le plus criant dans la zone qui nous intéresse est la région de Snåsa en actuelle Norvège. Le village same de Sørli, dont un habitant du nom de Oluf Jonsson payait l'impôt aux Norvégiens en 1548, se trouve sur les comptes de 1557 à 1559 à payer à la fois au lappfogde de Pite et au représentant du roi à Trondheim. Entre 1564 et 1570 a lieu la syvårkrig, et la situation devient encore plus complexe. En effet, les Suédois occupent le Jämtland et, en toute logique, y créent un lappfogde, dont le territoire n'est cependant pas entièrement clair. 
Tant et si bien que notre Oluf Jonsson se voit obligé pendant quelques années de payer un triple impôt! (Bergsland 1995, p. 43-45)

Dès lors, la situation se stabilise et, dans les zones disputées, la pratique montre que les Sames choisissent à qui ils veulent payer l'impôt. Or Gustav Vasa a eu l'idée efficace de nommer d'anciens birkarlar comme lappfogde. Ceux-ci sont plus attractifs aux yeux des Sames que les hommes du roi de Norvège. Devant ce constat, le slottsherr de Trondheim décide de reprendre les choses en main énergiquement. Il institue en 1591 un certain Torkil Nilsson comme "commandant des autres Lapons » dans la région de Snåsa, ayant tous pouvoirs pour commercer avec eux et lever l'impôt dessus : en d'autres termes, un lappfogde. De plus, il interdit sous peine de mort de payer un impôt à la Suède. L'efficacité de cette opération est discutable. Ironiquement, Torkil Nilsson luimême paye un impôt au lappfogde de Pite cette année-là. En 1603, lors de négociations à Flakkebæk, le roi de Norvège pousse ses revendications encore plus loin: il entend taxer l'ensemble des Sames de montagne, quel que soit le versant. Il n'abandonnera cette prétention qu'après sa défaite de 1645 (Bergsland 1995, p. 46-50). Une nouvelle tentative d'interdire de payer l'impôt aux Suédois est attestée en 1614, mais sans plus d'effet (Bergsland 1995, p. 52).

En 1606, le roi de Suède fait mener une série d'enquêtes, à la suite desquelles il décide de réformer le système d'imposition des Sames. En 1607, il crée le lappmark d'Ume renommé en lappmark de Lycksele en 1673 - qui inclut, outre les Sames de forêt des paroisses d'Umeå et Lycksele, les Sames d'Ångermanland, et ceux de toutes les zones doublement imposées, ce qui correspond à l'extrémité nord du Jämtland et aux régions de Bindal, Namdalen, et Snåsa en actuelle Norvège. Parallèlement, il augmente les impôts des Sames qui ne payent qu'à la Suède, et change en partie la nature de l'impôt : là où il exigeait uniquement des fourrures, il réclame désormais aussi des animaux vivants pour en faire des bêtes de trait (Bergsland 1995, p. 44-45, 50-51).

Les enquêtes du moment permettent d'avoir un aperçu de la situation économique des Sames du Sud à l'époque. On constate que l'élevage des rennes est encore très réduit : seuls les Sames de montagne le pratiquent, et les plus riches d'entre eux ne possèdent qu'une trentaine de bêtes. Cela change de manière assez brutale en 1620 et 1621. La mode à l'international a changé, et la demande en fourrures des pays occidentaux s'est écroulée. Il n'est dès lors plus intéressant d'imposer les Sames sous forme de fourrures, ni de faire du commerce avec eux sur ces mêmes biens. Les Sames du Sud n'ont alors plus rien à vendre qui intéresse les Scandinaves, et donc plus rien pour acheter un certain nombre de biens de première nécessité, comme des habits, du beurre ou de la farine. Ils se voient alors forcés de changer radicalement de mode de subsistance et basculent dans le pastoralisme, utilisant le renne majoritairement pour sa viande (Bergsland 1995, p.52). C'est à ce moment que commencent à apparaître les fattiglappar: ce sont des Sames ruinés qui, devenant parfaitement incapables de subvenir à leurs besoins, sont contraints de se placer sous la dépendance d'un autre Same qui, entre autres, paye l'impôt à leur place (Bergsland 1995, p. 61). Ce terme, jugé très insultant par les Sames, est encore de nos jours parfois utilisé par les Scandinaves pour parler d'eux 6 .

Une nouvelle guerre éclate en 1643, et elle touche cette fois de plein fouet les Sames du Sud : tous les chemins de montagne et de forêt entre le Bohuslän au sud et Snåsa sont occupés par des miliciens, ce qui a des conséquences évidentes sur l'économie des Sames (Bergsland 1995, p. 55). La paix est signée à Brömsebro le 23 août 1645 (n. s.), et 
la défaite de la Norvège est sévère : le Jämtland, le Härjedalen, et Älvdalen en Dalarna deviennent suédois (Kjersgaard 1982). Le Jämtland et le Härjedalen reçoivent leur propre registre d'imposition, où les Sames sont placés à part. La répartition interne qui en est faite montre vraisemblablement la répartition en sijth. On constate aussi à cette époque la paupérisation des Sames du Sud : un des deux Sames imposés dans la paroisse d'Offerdal disparaît des comptes en 1651, ruiné. Il ne payait déjà qu'un demi-impôt, preuve de sa faiblesse économique (Bergsland 1995, p. 57-58).

La Skånska krig, qui s'est déroulée de 1675 à 1679, touche durement la région des Sames du Sud. Les combats importants provoquent une famine en Jämtland, Härjedalen et Trøndelag. À la Noël 1676, le village de Sørli brûle, et, en 1678, c'est au tour de Røros (Bergsland 1995, p. 69). Quant aux Sames eux-mêmes, leurs ressources sont en grande partie détruites. Cas emblématique : le 7 février 1686, deux Sames se présentent au ting de Hildrem avec leur famille et leurs dépendants. Chacune de ces sijth possède environ 25 rennes, tout compris. Pour des gens qui, avant la guerre, en possédaient une centaine par sijte, le coup est difficile à encaisser, et ils viennent expliquer qu'ils ne sont plus en mesure de payer l'impôt (Bergsland 1995, p. 93) La Stor Nordiske krig, qui dure les vingt premières années du XVIII ${ }^{\mathrm{e}}$ siècle, achève de jeter un grand nombre de Sames dans la mendicité (Bergsland 1995, p. 69).

\section{Des paysans peu coopératifs} famille est massacrée par trois Scandinaves. Les coupables sont condamnés à mort en 1653, mais le mal est fait : la sijte qui occupait la montagne au nord de Särvan, dont Lars Klemetson était un dépendant, disparaît en 1656 (Bergsland 1995, p. 61). Et le cas n'est pas unique : en 1694, le ting de Snåsa condamne à mort les deux meurtriers de Blå-Nils, sa femme, ses six enfants et son dépendant. Sans aller forcément jusqu'au meurtre, les Scandinaves n'hésitent pas à se passer des services des tribunaux pour régler leurs conflits avec les Sames du Sud : en 1696, des paysans de Undersåker accusent un certain 
Torkel Olsson d'avoir causé des dégâts chez eux, et viennent lui prendre un renne en guise de réparation (Bergsland 1995, p. 89). Enfin, les paysans peuvent adopter une méthode plus subtile mais non moins efficace : ils installent une ferme en territoire same et attendent que les Sames partent d'eux-mêmes. À Kvelia, un Norvégien du nom de Per Påhlsson est venu installer en 1693 une ferme à côté du camp d'été d'un certain Thor Schiul; quelques années plus tard, il n'y avait plus de Sames à cet endroit. Pareillement, les Sames qui occupaient les forêts à proximité de Tydal ont été chassés par les paysans locaux en 1737, au motif fallacieux qu'ils étaient arrivés de plus au nord et qu'ils avaient fait disparaitre les rennes sauvages (Bergsland 1995, p. 91, 103-104). On voit ici apparaitre l'idée que les Sames du Sud, du moins ceux installés au sud de Frostviken, sont des immigrés récents: elle sera reprise, comme on l'a vu, par Yngvar Nielsen à la fin du XIX ${ }^{\mathrm{e}}$ siècle pour justifier le caractère allochtone des Sames en Norvège.

En Sør-Trøndelag, le recul des Sames est essentiellement dû à une découverte de 1631, et surtout 1644 : il y a du minerai de cuivre dans les montagnes au nord-est de Røros. Dès le 19 octobre 1646, Joachim Jürgens obtient un privilège royal sur le cuivre de la région, qui lui donne carte blanche pour exploiter les bois et les cascades. En conséquence, la zone, en quelques années, se peuple de villes, comme Brekken ou Kurås, et la forêt est presque entièrement rasée autour de Ålen, Haltdalen, ainsi qu'au sud de l'Aursunden. L'exploitation de Røros devient un véritable État dans l'État à la fin des années 1660, lorsque Joachim Jürgens obtient du roi le Helgeland avec presque tous les impôts sur les Sames, ainsi que de nombreux biens en Sør-Trøndelag, par l'intermédiaire d'un prête-nom (Bergsland 1995, p. 62-63).

\section{La christianisation des Sames}

La volonté de christianiser les Sames est ancienne : on en trouve les premières traces dès la fin $\mathrm{du}_{\mathrm{XIV}}^{\mathrm{e}}$ siècle, lorsque la reine de Suède en fait une nécessité. Sans succès. Bien au contraire, la fin du moyen âge voit une augmentation du nombre de tombes typiquement sames dans la zone same du Sud. Au XvII ${ }^{e}$ siècle, l'esprit missionnaire refait surface, et deux écoles sont créées, dont une à Lycksele, dans le but de former des prêtres sames qui puissent aller évangéliser les leurs (Hansen \& Olsen 2007, p. 319, 222). L'effet est à peu près nul. L'émergence du pastoralisme s'accompagne chez les Sames du Sud d'une ritualisation accrue des aspects religieux de la société. Ainsi, c'est à cette époque que l'on voit apparaître une division religieuse de la kåta : l'avant est profane, les femmes et le lait entrent par cette porte-là, tandis que l'arrière est sacré, c'est le territoire des hommes, et la porte de derrière sert à faire rentrer les armes et les animaux à sacrifier. C'est aussi à l'arrière que l'on entrepose le tambour chamanique (Hansen \& Olsen 2007, p. 232-233). Knut Bergsland relève qu'en 1663, au cours d'un procès de la région du Hommelfjell, des Sames manifestement baptisés - ils s'appellent Niels, Torkel, Jon, etc. - pratiquent encore une forme d'inhumation particulière, et rendent un culte à une divinité ours. De plus, les paysans locaux semblent parfaitement au courant de cet état de fait (1995, p. 65).

La question des noms est intéressante. En effet, Gustav Vasa a ordonné en 1559 la suppression des noms sames. Cela n'a pourtant pas suffi à décourager les Sames du Sud. Thomas von Westen (cf. plus loin) rapporte au début du XvIII siècle que les Sames ont une manière bien à eux de nommer les nouveau-nés. Un nom same lui est choisi au 
cours d'une cérémonie religieuse avant la naissance. Puis le nouveau-né est emmené à l'église pour être baptisé, et il reçoit alors un nom scandinave. Enfin, au cours d'une dernière cérémonie réalisée de retour chez eux, le bébé est débaptisé et rebaptisé de son nom same. En souvenir de cela, les Sames du Sud portaient tous un objet d'argent ou parfois de laiton, appelé un sjiele (Hansen \& Olsen 2007, p. 322-323). De nos jours, on peut choisir son nom librement, et pourtant, de nombreux Sames du Sud portent encore des objets en argent sur eux. Et il semble qu'ils leur accordent des vertus magiques, ou quelque chose s'en approchant: le mutisme de nos interlocuteurs ne nous a pas permis de creuser la question.

Paradoxalement, c'est de la mise en place de missions organisées au début du XVIII ${ }^{\mathrm{e}}$ siècle que va venir une amélioration - légère - de la condition des Sames en Norvège. En 1706, le roi Frederik IV envoie Povel Resen mener une enquête sur les Sames de son royaume. Celui-ci découvre que les fogden exercent une pression financière importante, trop importante même, sur les Sames, et que, de manière générale, la région est très mal connue des services centraux du royaume. Dès lors, le roi décide d'y mettre en place un représentant permanent de sa personne, avec pour mission de défendre aussi les intérêts des Sames. Le 10 décembre 1714, est créée la Finnemisjon, qui restera le principal organe de liaison avec les Sames de Norvège jusqu'en 1802 (Bergsland 1995, p.71-72). De cette commission est issu Thomas von Westen, un prêtre originaire de Trondheim. En 1722 et 1723, il a rendu visite à presque tous les groupes de Sames du Sud, dans le but de construire des églises et des écoles qui leur soient dédiées. Il rend compte à ses supérieurs que la religion chamanique est encore bien vivante, spécialement dans les montagnes autour de Namdalen, mais aussi à Meråker, par exemple. Il rend également compte de la profonde misère dans laquelle vivent la plupart d'entre eux. Aussi, dès 1724, il est décidé que les Sames du Sud ne paieront plus d'impôt. Et un rescrit royal de 1726 vise à leur rendre leurs anciens droits. Toutefois, l'efficacité de ces actions reste limitée: de nouveaux rapports des années 1730 et 1760 continuent à pointer du doigt la misère généralisée des Sames (Bergsland 1995, p. 98-99).

\section{Après la pluie vient le beau temps}

\section{Le Lappkodicill}

La Stor Nordiske krig a eu des conséquences assez dramatiques chez les deux belligérants, qui décident qu'une telle boucherie ne doit pas se reproduire. En 1734, après avoir contracté une alliance défensive, le Danemark-Norvège et la Suède conviennent qu'il serait temps de fixer précisément la frontière entre leurs deux États : seul le tronçon jusqu'au Dalsland était défini. Mais en 1737, il apparaît qu'il faut revoir l'ensemble de la frontière, et pas seulement les points de discorde. Au final, la Norvège accepte d'abandonner ses prétentions sur Frostviken, mais conserve Lierne, et la Suède abandonne ses propres prétentions sur Kautokeino, Karasjok et Utsjoki. Déterminer le tracé exact prend ensuite de longues années, et ce n'est qu'en 1749 que les plénipotentiaires peuvent se réunir à Strömstad pour régler les derniers points de détail. Enfin, le 21 septembre 1751 (n. s. ${ }^{7}$ ), le traité est signé. Là où ce traité intéresse directement l'histoire des Sames du Sud, c'est qu'il lui est adjoint un avenant, appelé couramment le Lappkodicill, qui est généralement considéré comme la première 
reconnaissance du peuple same comme un tout, indépendamment des frontières nationales (Gustafsson 1995).

L'article deux est tout d'abord une limitation : les Sames ne peuvent posséder des terres des deux côtés de la frontière. Pour le reste, il leur garantit un certain nombre de droits, quelle que soit leur nationalité. Les Sames ont d'ailleurs - partiellement - le droit de choisir cette nationalité, et leur neutralité est de toute manière garantie en cas de guerre entre les deux pays. De plus, leur droit d'élever des rennes est affirmé et, pour des raisons pratiques, ils ont également le droit d'utiliser la terre et l'eau de l'autre pays contre paiement d'une taxe modique : de nos jours, elle s'élève à 1 öre par tête de bétail. Enfin, en cas de conflit peu important entre Sames, ils ont le droit d'être jugés par leurs pairs ${ }^{8}$. Cette dernière disposition existait déjà en Norvège depuis 1707 (Bergsland 1995, p. 84).

\section{Fornorskning et forsvenskning}

49 J'ai choisi d'utiliser les termes norvégiens, plutôt que le terme français de norvégianisation, car ils ont un sens plus large : là où le français ne désigne que les actions politiques conscientes pour éliminer la langue et la culture sames, le norvégien englobe aussi toute les pressions économiques et culturelles venant par exemple de simples particuliers.

Les missions religieuses du xVIII siècle s'avèrent cette fois plutôt efficaces et, par conséquent, il apparaît désormais nécessaire de mettre en place un encadrement adéquat pour cette nouvelle population de croyants. Cela conduit à la création en Jämtland et Härjedalen d'une structure religieuse unique, les lappförsamlingar. Jusqu'à présent, les Sames d'une vaste zone étaient rattachés à une même paroisse : le Jämtland et le Härjedalen dépendaient tout entiers de la paroisse de Åsele, créée en 1648 à l'occasion du rattachement des deux provinces à la Suède. Mais en 1746, cela change : il est créé à Föllinge une paroisse uniquement dédiée aux Sames, et qui couvrait l'ensemble du territoire de ces provinces - son siège est transféré en 1852 à Hotagen. Quelques années plus tard s'en détache une nouvelle paroisse same, avec pour chef-lieu Undersåker, qui elle-même verra le Härjedalen lui échapper avec la création en 1768 du lappförsamling de Hede/Tännäs. Enfin, en 1842, c'est Frostviken qui se voit doté de sa propre paroisse 9 . Pour bien comprendre l'importance de ces créations, il faut se rappeler que les paroisses sont chargées de ce qui deviendra l'état civil; en d'autres termes, créer des paroisses parallèles réservées aux Sames, c'est faire de ceux-ci des sujets de seconde zone dès la naissance. Ce n'est qu'en 1942 que l'égalité religieuse avec les Scandinaves est obtenue avec l'abolition des lappförsamlingar.

51 Les attaques à l'encontre des langues sames sont particulièrement fortes en Norvège. En effet, lorsque ce pays se sépare du Danemark en 1814, naît un fort sentiment national, qui voit d'un mauvais œil l'existence de plusieurs langues et cultures au sein du pays. Aussi l'élimination va-t-elle être menée de manière consciente et organisée. Dans les modalités, le parallèle avec la politique d'élimination des langues régionales en France à la même époque est frappant. En 1880, les instructions officielles destinées aux professeurs indiquent que l'enseignement doit être fait en norvégien, et que le same ne sera utilisé que comme langue d'appoint, en cas d'incompréhension profonde. Puis à partir de 1905, lorsque la Norvège se sépare de l'union personnelle avec la Suède, cela monte d'un cran: l'usage du same est désormais interdit dans l'enseignement et dans le 
culte. L'élimination connaît un pic dans les années 1930, avec l'entrée dans les mentalités des Sames eux-mêmes de l'idée que leur langue n'a pas d'utilité, et qu'il est de meilleur goût de parler norvégien. Ce fut très efficace : entre 1930 et 1950, le same a presque entièrement disparu entre le Trøndelag et le Troms. Côté suédois, ce fut un peu moins brutal: il y a toujours eu un peu d'enseignement en same, notamment en Jämtland, et il y a même eu des tentatives de mettre en place des écoles itinérantes dont les professeurs suivraient les déplacements annuels de la communauté d'éleveurs.

D'un point de vue économique, la colonisation des territoires sames par les Scandinaves se poursuit : côté suédois, Gäddede et Jorm sont atteints dès le début des années 1760 ; côté norvégien, Åsmulen et Lassemoen sont occupés avant la fin du siècle et, en 1822, c'est toute la zone jusqu'aux lacs Namsvatnet et Tunnsjøen qui est colonisée. De plus, le XIX ${ }^{e}$ siècle entraîne le dernier changement économique profond des Sames du Sud : la présence de plus en plus nombreuse de Scandinaves les pousse à abandonner le troc et à rentrer dans une économie monétisée. La reconstruction après la seconde guerre mondiale porte un coup supplémentaire à l'élevage de rennes, car l'accent est alors essentiellement mis sur l'industrie et l'agriculture modernisée: le mode de vie des Sames est jugé trop archaïque pour mériter d'être aidé.

En ce qui concerne l'administration, les lappfogdar sont abolis en tant que tels en 1760 ; ils deviennent des fogdar comme les autres. Le titre est cependant recréé en 1885 en Jämtland, mais le lappfogde n'a plus désormais que des missions en lien avec l'élevage il est par exemple chargé de tenir le registre des marques de renne - et est chargé de la bonne entente entre Sames et sédentaires ${ }^{10}$. À partir de 1843, la Norvège commence à remettre en cause le Lappkodicill; en 1883, elle adopte la Felleskaploven, qui réduit les droits des Sames suédois en Norvège. Et la Suède accepte. Cette loi arrive à terme en 1907, mais la situation économique catastrophique en Suède du Nord dans les années 1910 pousse la Norvège à vouloir empêcher l'émigration de Sames suédois en Norvège. En 1919, les deux pays adoptent de concert la Renbeteskonvention, qui entre autres dispositions interdit aux Sames du Jämtlands län de traverser la frontière. La convention est modernisée en 1972, mais sans changement notable dans ses dispositions ${ }^{11}$.

\section{Une amélioration progressive}

L'amélioration des conditions économiques des sames de Suède commence en 1886 avec le renbeteslag : cette loi crée les lappby, entités administratives, économiques, et surtout collectives et territoriales chargées de la gestion de l'élevage des rennes par les Sames. En d'autres termes, il est reconnu aux Sames le droit d'élever leurs rennes sur un territoire qui, sans leur appartenir, ne peut être colonisé par des Scandinaves. La loi est révisée en 1928 pour y intégrer une définition précise de qui est same, ainsi que pour supprimer l'impôt particulier qui touchait les Sames : ce sont désormais des citoyens comme les autres aux yeux de l'administration fiscale. En 1971, enfin, le renbeteslag est remplacé par le rennäringslag, qui d'une part renomme les lappbyar en samebyar - l'autoethnonyme du peuple est enfin utilisé légalement - et, d'autre part accorde aux samebyar et à eux seuls le droit de pratiquer l'élevage de rennes. Cette nouvelle loi supprime également le lappfoged de Jämtland : les questions de rennes sont directement gérées par la commission aux affaires agricoles du länsstyrelse. Enfin, en 2006, le pouvoir de créer ou modifier un sameby est transféré du länsstyrelse au Sametinget. 
Pour terminer sur l'histoire du xxle siècle, la convention de 1972 est arrivée à expiration en mai 2005, et les deux gouvernements travaillent à en trouver une nouvelle. Mais la Norvège veut limiter autant que possible les droits des Sames de Suède sur son territoire, ce qui rend les négociations difficiles. Un reportage d'Ođđasat ${ }^{12} \mathrm{du}$ 20 septembre 2011 expliquait qu'aucun accord n'avait encore pu être trouvé, et que les autorités espéraient y parvenir à l'été 2012.

\section{Conclusion}

Comme on a pu le voir, la connaissance de l'histoire des Sames avant le xviE siècle est en pleine évolution et, pour cette raison même, de nombreux points en sont encore disputés. Mais il ne fait aucun doute que les questions finiront par être tranchées, la communauté des linguistes, archéologues et historiens qui s'y intéressent étant fort dynamique. Il est cependant un domaine qui nécessiterait encore d'être exploré ; c'est l'histoire récente des Sames du Sud : en effet, il est actuellement très difficile - en ne se fondant que sur des publications déjà existantes - de rédiger une histoire des Sames du sud après 1750 qui soit clairement distincte d'une histoire générale des Sames.

\section{BIBLIOGRAPHIE}

AIKIO Ante, 2006, “On Germanic-Saami contacts and Saami prehistory”, Journal de la Société Finno-

Ougrienne, 91, p. 9-55.

BERGSLAND Knut, 1995, Bidrag til sydsamenes historie, Skriftserie - Senter for samiske studier, 1, Tromsø, Universitetet i Tromsø.

GUSTAFSSON Åke, 1995, Riksgränshistoria och gränsöversyner, Gävle, Lantmäteriet.

HANSEN Lars Ivan, oLSEN Bjørnar, 2007, Samenes historie fram til 1750, Oslo, Cappelen akademisk forlag.

INGMAN Max, GYLLENSTEN Ulf, 2007, “A recent genetic link between Sami and the Volga-Ural region of Russia", European Journal of Human Genetics, 15, p. 115-120.

KALLIO Petri, 2009, “Stratigraphy of Indo-European Loanwords in Saami” in ÄIKÄs Tiina (éd.), Máttut - máddagat: The Roots of Saami Ethnicities, Societies and Spaces / Places, Oulu, Giellagas Institute, 2009, p. 30-45.

KARISSON Andreas et al., 2006, "Y-chromosome diversity in Sweden - A long time perspective", European Journal of Human Genetics, 14, p. 963-970.

KJERSGAARD Erik, 1982, Kjersgaards Danmarkshistorie, T. 2, København, Komma.

ROOTSI Siiri et al., 2007, "A counter-clockwise northern route of the Y-chromosome haplogroup N from Southeast Asia towards Europe”, European Journal of Human Genetics, 15, p. 204-211. 
STRADE Norbert, 1997, "Det sydsamiske sprog” in ZACHRISSON Inger (éd.), Möten i gränsland : Samer och germaner i Mellanskandinavien, Stockholm, Statens historiska museum, 1997, p. 175-185.

WELINDER Stig, 2008, Jämtarna och Samerna kom först, Östersund, Jamtli förlag.

\section{NOTES}

1. La rédaction de cet article fait suite à une expédition en Jämtland menée conjointement par cinq étudiants de l'INALCO pendant l'été 2010. Nous avions pour ambition principale de récolter des enregistrements audio en same du sud auprès de locuteurs locaux, et de prendre des contacts pour une éventuelle future expédition. C'est pour cela que l'expédition est mentionnée aux notes 3 et 6 comme un fait acquis. La publication d'un recueil commun d'articles, prévue à l'origine, n'a malheureusement pas pu se faire.

2. Publié par l'Institut finnois de recherche en linguistique et disponible à l'adresse http://kaino.kotus.fi/algu/

3. Montagne au nord-ouest de Funäsdalen. Juste en face, de l'autre côté de la vallée, se trouve le village same de Mittåhammaren, que notre expédition a visité.

4. Chaser un esclave : terme plutôt employé pour l'histoire de France; cela consiste à affranchir un esclave pour lui faire exploiter en métairie une terre qui jusqu'alors était exploitée directement par son propriétaire.

5. Informations tirées de l'inventaire du landsarkiv $i$ Östersund disponible à l'adresse http://nad.ra.se/

6. Communication orale de Sarah A., jeune fille same du Sud que nous avons rencontrée lors de notre voyage.

7. D'après le calendrier grégorien.

8. Le détail de ces dispositions est disponible sur le site officiel du Samiskt Informationscentrum, un service du Sametinget : http://www.samer.se/1601

9. Informations tirées de l'inventaire du landsarkiv $i$ Östersund.

10. Informations tirées de l'inventaire du landsarkiv $i$ östersund

11. Informations tirées du site officiel du Samiskt Informations-centrum: http:// www.samer.se/1601

12. Ođđasat est une émission d'informations en same du Nord diffusée sur les chaînes nationales des trois pays.

\section{RÉSUMÉS}

Les publications récentes en langue française sur les Sames ne manquent pas, qu'il s'agisse de linguistique, d'ethnologie ou d'œuvres littéraires traduites. En revanche, au cours des dix dernières années, l'histoire a été entièrement délaissée : seul Christian Mériot a publié en 2001 
une étude ethnologique de la vision qu'ont les Sames de leur histoire. Pourtant, de nombreux chercheurs, norvégiens, suédois et finlandais ont fait considérablement avancer la science depuis quinze ans. On est en droit de dire qu'ils ont révolutionné notre connaissance de l'histoire des Sames. Mais leurs ouvrages sont au mieux difficilement accessibles, au pire parfaitement introuvables en France. Aussi me suis-je décidé à faire un rapide aperçu de l'état de la science. Le but n'est pas d'être exhaustif, mais de mettre en lumière les fils conducteurs qui sous-tendent l'histoire des Sames du Sud. On pourra ainsi apprendre, entre autres choses, que les Sames ne sont pas le peuple autochtone de la péninsule scandinave, qu'ils ne pratiquaient presque pas d'élevage du renne avant le XVII ${ }^{\mathrm{e}}$ siècle, qu'il est impossible de comprendre leur histoire entre le $\mathrm{XVI}^{\mathrm{e}}$ et le $\mathrm{XVIII}^{\mathrm{e}}$ siècles sans la remettre dans le contexte des conflits de frontière suédonorvégiens, et que l'histoire récente des Sames du Sud proprement dits est mal connue.

Recent publications in French about the Saami aren't uncommon, be it linguistics, ethnology or translated literature. However, during the last ten years, history has been thoroughly neglected: only Christian Mériot published an ethnologic survey of Saami vision of their history, in 2001. Yet, numerous Norwegian, Swedish or Finnish researchers have lately been greatly improving the science. We can even say that they have revolutionised our knowledge of Saami history. But their works are at best not easily accessible in France. Hence I made my mind up to produce a quick overview of the current state of the art. The goal is not to be comprehensive, but to point the underlying guidelines of South Saami history out. We will therefore learn, amidst other things, that the Saami are not the aboriginal people of the Scandinavian Peninsula, that they almost didn't use reindeer herding before the $17^{\text {th }}$ century, that their history between the $16^{\text {th }}$ and $18^{\text {th }}$ centuries is incomprehensible without putting it in the context of Swedish-Norwegian border conflicts, and that the recent history of the South Saami proper is scarcely known.

De senare publiceringarna i franska om samerna saknas inte, antingen språkvetenskap, etnologi eller översatt litteratur. Däremot, under de senaste tio åren, historien har helt övergetts : bara Christian Mérot publicerade 2001 en etnologiske studie om hur samerna ser sin egen historia. Ännu, många norska, svenska eller finska forskare har starkt förbättrat vetenskapen sedan femton år. Det kan rimligtvis sägs att de har revolutionerat vår kunskap av samernas historia. Men deras böckerna är i bästa fall svår tillgängliga, i värsta helt ofinnbara i Frankrike. Så jag bestämde mig för att utgiva en snabb översikt över vetenskaps nuvarande staten. Målet är inte att vara omfattande, utan att föra sydsamernas historias huvudaxlarna i ljuset fram. Det kan således läras sig, bland annat, att samerna är inte ursprungsfolket av den skandinaviska halvön, att de hade närmare ingen tamren före 1600-talet, att det är omöjligt att förstå deras historia mellan 1500- och 1700-talet utan att överväga kontexten av svensk-norska gränsstrider, och att sydsamernas senaste historia är oklart. 


\section{INDEX}

Keywords : hunting and fishing husbandry, pastoralism, Ethnicity, reindeer herding, ethnic conflicts, funerary customs, tax policy, Colonisation, Christianisation, history, Finns, Germanic peoples, Mari, Northern Saamis, Saamis, Scandinavians, Southern Saamis, Swedes, Volga Bulgars, Finnish, Norwegian, Old Norse, Proto-Fennic, Proto-germanic, Proto-Saami, Proto-Scandinavian, Southern Sami

Index chronologique : préhistoire, paléolithique, VIe siècle, VIIe siècle, VIIIe siècle, Xe siècle, XIe siècle, XIIe siècle, XIVe siècle, XVe siècle, XVIe siècle, XVIIe siècle, XVIIIe siècle, XIXe siècle Mots-clés : économie de chasse et de pêche, Stor Nordiske krig, économie pastorale, ethnicité, Sjiele, élevage du renne, Samebyar, conflits ethniques, Rennäringslag, coutumes funéraires, Fornorskning, fiscalité, colonisation, Forsvenskning, christianisation, Ođđasat, Lappkodicill, Lappby, Lappfogdar, Lappförsamlingar, Fattiglappar, Länsstyrelse, Felleskaploven, Birkarlar, Renbeteskonvention, Sametinget, Skånska Krig, Ting, Vuopmanat

nomsmotscles Sames du sud, Finlandais, Scandinaves, Suédois, Sames, Maris, Germains, Bulgares de la Volga

disciplines proto-scandinave, proto-same, proto-germanique, proto-fennique, same du sud, norvégien, norrois, finnois

Index géographique : Aursunden, Ålen, Åsele, Åsmulen, Älvdalen, Ångermanland, Bindal, Bohuslän, Brandval, Brekken, Brömsebro, Cap Nord, Dalsland, Danemark, Duved, Femunden, Finlande, Finnfjellet, Flakkebæk, Föllinge, Frostviken, Funäsdalen, Gäddede, Gjevsjø, golfe de Botnie, golfe de Finlande, Haltdalen, Hamar, Hålogaland, Härjedalen, Hedmark, Helgeland, Hildrem, Hommelfjell, Hotagen, Jämtland, Jorm, Karasjok, Kautokeino, Kjelmøy, Kolåsen, Kvelia, Ladoga (lac), Lassemoen, Långön, Lierne, Ljungdalen, Lule, Lycksele, Medelpad, Meråker, Mittåhammaren, Namdalen, Namsvatnet, Njaarke, Norvège, Novgorod, Nyamočokka, Offerdal, Onega, Oslo, Östersund, Ostrobotnie, Oural, Øvre-Rendalen, Røros, Pays basque, Pite (rivière), Rana, Fédération de Russie, Savo (île), Särvan, Scandinavie, Sibérie, Snåsa, Sør-Trøndelag, Sösjön, Sørli, Stor-Mittåkläppen, Suède, Storsjö, Strömstad, Strömsund, Sunne, Tampere, Troms, Trøndelag, Trondheim, Tunnsjøen, Tynset, Umeå Ume, Undersåker, Uppsala, Utsjoki, Verdal, Vivallen, Volga (vallée de la)

Thèmes : histoire 